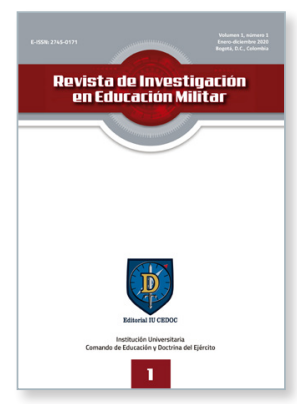

Revista de Investigación en Educación Militar

Volumen 1, número 1, enero-diciembre 2020, pp. 51-68

Bogotá, D. C., Colombia

E-ISSN: 2745-0171

https://doi.org/10.47961/27450171.5

\title{
Relaciones entre Colombia y sus vecinos frente a la ayuda internacional
}

\section{Robert Manuel Ojeda Pérez}

https://orcid.org/0000-0002-1227-7854

rojeda@unisalle.edu.co

Universidad de La Salle, Bogotá D. C., Colombia

\section{Elizabeth Martínez Cruz}

https://orcid.org/0000-0003-0110-092X

emartinez17@unisalle.edu.co

Universidad de La Salle, Bogotá, D. C., Colombia

Citación: Ojeda Pérez, R. M., \& Martínez Cruz, E. (2020). Relaciones entre Colombia y sus vecinos frente a la ayuda internacional. Revista de Investigación en Educación Militar, 1(1), 51-68. DOl: https://doi.org/10.47961/27450171.5

Publicado en línea: $1 .^{\circ}$ de diciembre de 2020

Los artículos publicados por la Revista de Investigación en Educación Militar son de acceso abierto bajo una licencia Creative Commons: Atribución - No Comercial - Sin Derivados.

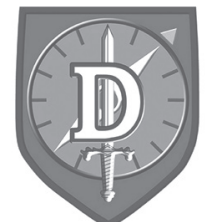

Editorial IU CEDOC 


\title{
Relaciones entre Colombia y sus vecinos frente a la ayuda internacional
}

Relations between Colombia and its neighbors in the face of international aid

\author{
Robert Manuel Ojeda Pérez \\ Elizabeth Martínez Cruz \\ Universidad de La Salle, Bogotá D. C., Colombia
}

\section{Resumen}

Este artículo analiza los impactos del Plan Colombia en las relaciones de Colombia con los países vecinos. Con este objetivo, se hace una revisión documental con la metodología del marco analítico de Charles Tilly para analizar el punto de vista de los gobiernos de la región sobre sus relaciones con Colombia y determinar las consecuencias de la ayuda de Estados Unidos en las negociaciones del Tratado de Libre Comercio y en la soberanía colombiana. Los resultados del análisis muestran que la alianza militar fortaleció las relaciones entre Colombia y Estados Unidos, pero deterioró las relaciones con los países vecinos debido a que la presión del gobierno estadounidense generó el desplazamiento de los grupos al margen de la ley y acciones militares de Colombia fuera de sus fronteras.

Palabras clave: alianza militar; ayuda internacional; grupos al margen de la ley; países vecinos; Plan Colombia.

\section{Abstract}

This article analyzes the impacts of Plan Colombia regarding Colombia's relations with neighboring countries. With this objective, a documentary review is made with the methodology of the analytical framework of Charles Tilly to analyze the point of view of the governments of the region on their relations with Colombia and determine the consequences of the aid coming from the United States in the negotiations of the Free Trade Agreement and in Colombian sovereignty. The results of the analysis show that the military alliance strengthened relations between Colombia and the United States, but deteriorated relations with neighboring countries due to pressure from the United States government causing the displacement of illegal groups and Colombian military actions outside its borders.

Keywords: international aid; groups outside the law; military alliance; neighbor countries; Plan Colombia 


\section{Introducción}

Este artículo aborda las relaciones internacionales entre Colombia y sus países vecinos frente a la ayuda internacional en el Plan Colombia, que se dio en el mandato del expresidente Álvaro Uribe Vélez (2002-2006, 2006-2010) con el presidente de Estados Unidos George Walker Bush (2001-2009). Esta relación fue relevante en la política exterior del gobierno del presidente George Walker Bush con América Latina debido a que los vínculos forjados con Colombia tuvieron notoriedad mundial (Tickner, 2008). Por su parte, la Presidencia de Colombia enfocó su agenda internacional en la relación con los Estados Unidos, especialmente en la estructuración y funcionamiento del Plan Colombia debido al importante apoyo que le brindó el gobierno norteamericano, un proceso que ha logrado avances a través de diferentes estrategias (Rojas, 2006).

Los acercamientos entre los dos gobiernos les permitieron tratar los temas más relevantes, como el problema de las drogas para el gobierno colombiano, lo cual condujo a que tuviera una relación estrecha con los Estados Unidos debido a que en ese momento sostenía una guerra contra el terrorismo en la esfera internacional (Tickner, 2008). De esta manera, Colombia entendió que era importante crear un vínculo con los estadounidenses de cualquier manera que fuera posible, incluso plegándose a adoptar una agenda internacional basada en el ataque al terrorismo, como se verá a continuación.

Como respuesta a estas problemáticas — que existían desde un tiempo considerable antes de los gobiernos de Uribe y Bush—, en 1999 se definió el Plan Colombia durante el mandato de los presidentes Andrés Pastrana (1998-2002) y Bill Clinton (1993-2001), quienes tenían unos objetivos específicos en este proyecto de seis años: "Generar una revitalización social y económica, terminar el conflicto armado en Colombia y crear una estrategia antinarcóticos" (Veillette, 2005, p. 1).

Este documento se fundamenta en la posición que Charles Tilly (2007) desarrolla en "Guerra y construcción del Estado como crimen organizado". En ese texto argumenta que los Estados que surgen a través de cualquier actividad, ya sea la guerra, la protección o extracción, afecta posteriormente su propio desarrollo y les impide un equilibrio, ya que la guerra es una condición natural para defender su posicionamiento dentro del sistema internacional. Del mismo modo, al ser Colombia un Estado dependiente de un actor externo, en la medida en que intercambia material y destreza militar por materias primas o personal de apoyo, aquella influencia externa ejerce un elevado poder sobre las organizaciones del Estado y su desarroIlo, entre las cuales se encuentran las encargadas de salvaguardar las fronteras (Tilly, 2007).

Con base en estos postulados, se puede afirmar que Estados Unidos es una organización que busca aliados estratégicos en contra del terrorismo, perspectiva que, como veremos, también fue acogida por el gobierno de Uribe. Uno de los principales objetivos del expresidente Uribe en la diplomacia nacional era lograr que las Fuerzas Armadas Revolucionarias de Colombia (FARC) fueran consideradas como un grupo terrorista internacional, con lo cual conseguía 
que estuvieran en el listado de organizaciones terroristas del Departamento de Estado. De esta manera, no se les permitiría el ingreso a espacios internacionales y las finanzas de origen ilegal tendrían un seguimiento. Asimismo, las autoridades podrían usar la extradición para ejercer una amenaza contra los guerrilleros, no solamente por el tráfico de drogas, sino también por participar en actividades terroristas y obstruir la guerra contra las drogas, que era la política exterior estadounidense en ese momento (Rojas, 2006).

Teniendo en cuenta los postulados de Tilly (2007), Uribe amenazaba con hacer la guerra a las FARC, las cuales no solamente atentaban contra el Estado colombiano, sino también contra los intereses del gobierno norteamericano, así que fue necesario fortalecer las Fuerzas Militares para que pudieran brindar protección a la soberanía nacional.

A partir de lo anterior surge una problemática que se refleja en el momento en que el Estado colombiano no tiene el control del territorio de forma soberana, debido a la creación y evolución de grupos al margen de la ley, los cuales violaron los Derechos Humanos en diferentes regiones del país y expandieron el narcotráfico alrededor del mundo. Por lo tanto, para fortalecer el Estado había que amenazar con la guerra, pero al buscar la ayuda internacional pudo haber perdido la soberanía y abrir las posibilidades para la extracción de recursos en el territorio, elementos que se estudian en este documento.

La ayuda de Bush a Colombia fue percibida por los países vecinos como una amenaza en el equilibrio territorial a causa de la lucha contra las drogas ilícitas, que generó fenómenos como desplazamiento, narcotráfico e incursiones guerrilleras, lo cual repercutió directamente en los Estados vecinos. De igual modo, la relación entre los gobiernos de Uribe y Bush también generaron una incertidumbre en los países vecinos, pues, como potencia mundial, los Estados Unidos podrían tener intención de intervenir en los territorios latinoamericanos a través del fortalecimiento de las Fuerzas Militares colombianas — tanto en estrategia como en arsenal - y por medio de la tecnología y la información que le están suministrando a cambio de la extracción, como por ejemplo las negociaciones de los tratados de libre comercio. Es decir, se percibe que Estados Unidos tiene el papel de mayor autoridad por ser la potencia mundial, provee protección a Colombia y debido a la supremacía que ejerce, los rivales se ven obligados a ceder a su potestad (Rojas, 2006).

En este sentido, un tema relevante son las acciones que el presidente Uribe realizó para convertirse en un aliado permanente del país norteamericano. Desde una perspectiva schmittiana, esto se comprende en la distinción entre amigo o enemigo, como lo anunció el presidente Bush luego del ataque terrorista a las Torres Gemelas el 11 de septiembre de 2001: "0 se está con nosotros, o contra nosotros" (Rojas, 2006).

Esto evidencia que Colombia estuvo condicionada desde el primer momento a repartir el poder militar con los Estados Unidos, ya que los grupos al margen de la ley, especialmente las FARC, tenían el monopolio de la violencia y el paramilitarismo había surgido. Esta situación 
produjo que el Estado perdiera la soberanía y cediera a la ayuda internacional con todas las condiciones impuestas por parte del país norteamericano. Es decir, Colombia optó por "hacer la guerra para construir el Estado, pero permitiendo su extracción" (Tilly, 2007, p. 24).

\section{Variables problemáticas desde el postulado de Tilly}

En el documento se desarrollan tres problemáticas que surgieron a partir de las negociaciones del Plan Colombia entre el presidente de Colombia, Álvaro Uribe Vélez, y el presidente de los Estados Unidos, George W. Bush.

La primera problemática fue el deterioro de las relaciones de Colombia con sus países vecinos como consecuencia de la presión que los Estados Unidos ejercieron sobre el país para obtener resultados en el Plan Colombia. Esta situación desencadenó principalmente una fuerte enemistad con Ecuador y Venezuela, además de que impactó las relaciones internacionales con varios países de Centro y Suramérica, así como con Estados de la Unión Europea. Sin embargo, a la vez Colombia estableció una cooperación con Perú y Bolivia en la lucha contra el narcotráfico.

La segunda problemática tiene que ver con las decisiones que se tomaron en la negociación del Tratado de Libre Comercio (TLC) y la posición que asumieron los estadounidenses frente al Plan Colombia. Finalmente, la tercera problemática se refiere a la manera como la alianza militar fortaleció las relaciones entre estos dos países, pero permitió la extracción mediante los tratados comerciales.

A partir de estas problemáticas surge la pregunta de investigación: ¿Cuál fue el impacto que tuvo la ayuda internacional de los Estados Unidos con el Plan Colombia en las relaciones internacionales entre Colombia y los países vecinos? En este sentido, el objetivo general del estudio es analizar tanto los aspectos que funcionaron, como los que fueron cuestionados en los campos políticos, económicos y sociales en la relación con los países vecinos Ecuador, Venezuela, Perú y Bolivia entre los años 2001 y 2009.

La hipótesis de este trabajo se basa en la ecuación propuesta por Tilly (2007), según la cual la guerra genera el Estado y desde alli es posible tanto la extracción como el crimen organizado. Esta ecuación se emplea para analizar la relación entre Colombia y un aliado como Estados Unidos en torno al Plan Colombia, que se originó a causa de la confrontación armada y la corrupción por parte del ataque al narcotráfico. Esta situación debilitó las instituciones gubernamentales y generó una desconfianza entre los países vecinos debido a la alianza con Estados Unidos, que presionaba por resultados para cumplir el Plan Colombia.

Para cumplir este objetivo de investigación, se identifican los impactos del Plan Colombia en las relaciones de Colombia con Venezuela, Perú, Ecuador y Bolivia, así como los argumentos de estos países frente a la ayuda internacional estadounidense. A su vez, se estudia la toma de decisiones en el Tratado de Libre Comercio y las diferentes visiones 
que tuvo Estados Unidos respecto al Plan Colombia. Por último, se indaga de qué manera la alianza militar fortaleció los vínculos entre Colombia y los Estados Unidos.

Como se había mencionado, el principal referente de análisis en este artículo es el trabajo de Charles Tilly (2007) titulado "Guerra y construcción del Estado como crimen organizado", donde plantea que "el negocio de la protección representa el crimen organizado en su versión más sofisticada" (Tilly, 2007, p. 1). Específicamente, Tilly (2007) argumenta que los agentes del Estado incluyen cuatro actividades diferentes: (1) la guerra como medio para neutralizar o eliminar a los adversarios en un espacio donde estos carezcan de ventajas, primordialmente en un territorio desconocido, de tal forma que se reduce su uso de la fuerza; (2) la construcción del Estado, en la medida en que se pueden eliminar o neutralizar adversarios dentro de la región para dar origen a un nuevo Estado; (3) la protección, que se logra eliminando o neutralizando a los adversarios de los clientes y, por último, (4) la extracción, que hace referencia a la obtención de diferentes recursos que generen una utilidad para poder realizar las actividades de guerra, construcción del Estado y protección (Tilly, 2007).

Además, el segundo referente es la teoría neoliberal, que propone a los gobiernos de diferentes países la apertura de sus mercados como una medida para fomentar el comercio nacional hacia una libre competencia. Sin embargo, estas medidas afectan en la práctica a los países en desarrollo, así que implementan unas estrategias en su política exterior que les garanticen estabilidad en el comercio internacional a través de la constitución de alianzas (Tilly, 2007). Esto les permite eliminar barreras comerciales mediante la creación de TLC, como es el caso de Colombia con Estados Unidos, un punto importante que refleja el apoyo que dieron los estadounidenses a Colombia con la ayuda internacional.

\section{Plan Colombia, terrorismo y narcotráfico}

En la década del noventa Colombia presentaba una serie de problemáticas, principalmente debido a la intensificación del conflicto armado. Esta situación era generada por el notorio crecimiento estratégico de las FARC, el cual no solo acarreaba el aumento de la violencia, sino además la propagación de la plantación de hoja de coca, que contribuía al masivo ingreso de dinero ilícito y que dejaba a Colombia como uno de los principales mercados de coca en el mundo. Según el Comité Interinstitucional de Lucha contra las Finanzas de Subversión (Gutiérrez, 2015), "en 1994 las guerrillas estaban recibiendo 219 billones de pesos por concepto de narcotráfico, y 685 en 1996" (p. 18).

Esto explica la ineficacia del gobierno colombiano en la terminación del conflicto, así como su debilidad frente al sistema internacional, que se constata en los mandatos de GuiIlermo León Valencia (1962-1966), Belisario Betancur (1980-1984), César Gaviria (19901994) y Andrés Pastrana (1998-2002), los cuales realizaron algunos diálogos con la guerrilla, pero no los materializaron (Gutiérrez, 2015). En este difícil contexto, las Fuerzas Armadas 
pierden el control de la protección a la población colombiana, de modo que surge la necesidad de recibir la ayuda internacional estadounidense para contrarrestar la violencia ejercida por las guerrillas (Tickner, 2008).

El fenómeno del narcotráfico, que ha sido uno de los temas más importantes de la agenda internacional de los Estados Unidos, ha tenido una importante evolución, que inicia con la aparición de plantaciones de coca en Colombia, luego de que Perú y Bolivia fueran las bases principales de la producción en Latinoamérica. Posteriormente, por una parte, surgen las plantaciones de amapola en los departamentos de Tolima y Huila, y, por otra, la guerrilla se convierte en un cartel, lo cual genera una lucha de carteles por el poder sobre rutas y mercados. La consolidación económica que produjo este proceso permitió el desbordamiento de poder, que se reflejó en la corrupción, el poder armado y la obtención de curules en el Congreso por parte de capos, lo cual llevó a que una gran parte de políticos estuviera a su favor y beneficio (García, 2013).

En respuesta a esta problemática se formuló el Plan Colombia, en el cual el gobierno colombiano se impuso en el año 2000 invertir en el campo social y acabar los cultivos de coca, así como desmovilizar las guerrillas, mientras que los Estados Unidos se concentraban principalmente en derrocar el narcotráfico.

El desacuerdo y la cooperación entre los países vecinos Colombia-Ecuador generaban una tensión negativa en sus relaciones debido al Plan Colombia. Esta disconformidad se evidenció en los Libros Blancos del Ministerio de Defensa del Ecuador (2000-2006), donde acusaba al Plan Colombia como una amenaza "con formas de violencia que hoy se presentan como enfrentamientos globalizados, sin fronteras, en los que no necesariamente intervienen fuerzas armadas organizadas y con la población civil como blanco" (Aranguren, 2013, p. 9).

Las consecuencias del narcotráfico y el poder que ejercían las FARC en el territorio colombiano creaba problemas en la seguridad de Ecuador, que se pueden sintetizar en cuatro situaciones principales (Aranguren, 2013): (1) la presencia de redes de tráfico transnacional de drogas en su territorio, (2) la presencia en la frontera de grupos de la guerrilla, (3) el daño que producen las fumigaciones con glifosato al medio ambiente y la intervención de los Estados Unidos, y (4) el aumento de refugiados y desplazados colombianos en el vecino país.

La descripción de estas problemáticas demuestra a primera vista que Ecuador ya planteaba una oposición respecto a la ayuda internacional del Plan Colombia. Esta percepción se justificaba en la posible expansión del narcotráfico existente en Colombia y sus consecuencias en el territorio ecuatoriano, así como en el temor a una posible intervención por parte de la gran potencia, Estados Unidos, en su territorio.

Por el contrario, Colombia estableció una relación de cooperación en la lucha contra el narcotráfico con Perú y Bolivia con el fin de recibir los recursos que ofrecían los Estados Unidos a través de su política antinarcóticos. Estos recursos económicos lograrían una moder- 
nización de las entidades gubernamentales que se encargan de disminuir las plantaciones de coca en cada territorio. Sin embargo, la presencia del Plan Colombia en 1998 empeoró la situación de las fronteras amazónicas con Venezuela, Brasil, Perú y Ecuador debido a que fabricantes de droga y grupos al margen de la ley, como las guerrillas y los paramilitares colombianos, se desplazaron a estas zonas marginadas que no cuentan con buena financiación del Estado, lo cual agravó el conflicto interno (Moreano, 2005).

Por lo tanto, Bolivia y Perú consideraron el desarrollo del Plan Colombia como una forma de protección y de guerra para acabar no solo con los productores de droga, sino también con el monopolio de la violencia que tenían los guerrilleros y los paramilitares colombianos que amenazaban sus fronteras. No obstante, les beneficiaría también los recursos que les concedían los estadounidenses para mejorar sus organizaciones estatales contra la droga y hacer nuevas propuestas dirigidas a los cultivadores de la planta de coca en sus regiones, con la finalidad de empezar un cambio en sus zonas de conflicto erradicando la materia prima para el negocio del narcotráfico.

\section{Presiones del Plan Colombia}

Entre las tensiones que generó el Plan Colombia se encuentra, además, una crisis diplomática entre Colombia y Ecuador que surgió el 29 de febrero de 2008 en territorio ecuatoriano. Como afirma Cortés y Águila (2012), la crisis diplomática se originó porque las Fuerzas Armadas colombianas bombardearon un campamento guerrillero de las FARC sin dar un aviso previo a las Fuerzas Militares ecuatorianas. Este bombardeo tuvo lugar en la provincia de Sucumbíos y ocasionó la muerte de Raúl Reyes, el segundo al mando de este grupo guerrillero, de 18 guerrilleros y de cuatro estudiantes mexicanos. Debido al ataque sin autorización, el gobierno de Ecuador acusó a Colombia por la violación a su soberanía y el incumplimiento de la Convención de Viena de 1961.

Esta acción refleja el factor de guerra expuesto por Tilly. En este caso, Estados Unidos como potencia mayor neutraliza a los rivales, que se encuentran fuera del territorio de preferencia. De esta forma, además, presiona a Colombia por medio del uso de la fuerza, como fue el apoyo militar en el bombardeo al campamento de las FARC en territorio ecuatoriano.

Rafael Correa, a la vez, se acercó a las instituciones internacionales para impedir que el conflicto en Colombia fuera externalizado. Entre las medidas que tomó el mandatario se encuentra el desplazamiento de tropas en la frontera del norte que logró con la junta del Consejo de Seguridad Nacional, además habló con la Comunidad Andina de Naciones (CAN), el Mercosur y el Consejo Permanente de la Organización de Estados Americanos (OEA), donde expresó la pretensión de que el gobierno colombiano ofreciera una disculpa y se comprometiera frente a la presencia de organizaciones internacionales, además de que garantizara que no volvería a reiterar un hecho de esta magnitud, que consideró como originado por la violencia colombiana y la política del mandatario Álvaro Uribe Vélez (Lamrani, 2008). 
Estos llamados a entes internacionales fueron tomados como una represalia del presidente Correa porque sintió que Colombia había afectado su soberanía. Además, posteriormente también tomó la decisión de retirar al embajador colombiano en su país, Carlos Holguín, lo cual demuestra la fuerte crisis diplomática que tuvieron estas dos naciones.

En este contexto, debido a la acción militar de Colombia en Ecuador, Venezuela decidió apoyar a los ecuatorianos. El 2 de marzo del 2008 el presidente venezolano Hugo Chávez movilizó tropas a la frontera, acusó a Colombia de ser "un Estado terrorista" y advirtió a su homólogo colombiano, Álvaro Uribe: "Si por ejemplo a usted se le ocurre hacer eso en Venezuela, le mando unos Sukhois (aviones cazas de fabricación rusa), compañero, para que lo sepa" ("Venezuela y Colombia....", 2010). No solamente Ecuador tomó represalias contra Colombia, sino que también lo hizo Venezuela, al sentir que la ayuda internacional ofrecida por Estados Unidos mediante el Plan Colombia representaba una amenaza para su gobierno.

Por tal motivo, el mandatario Hugo Chávez acusó a Colombia por su política de terrorismo e hizo un llamado a todos los gobiernos de América Latina para que tomaran medidas frente a esta situación de terrorismo colombiano, que en su criterio estaba afectando a todos los países vecinos. Chávez se dirigió a Uribe con expresiones severas: " 'No solo es un lacayo del imperio, no solo es un mentiroso, es un criminal, un mafioso. Dirige un narcogobierno, un gobierno paramilitar', en referencia a los vínculos notorios que tiene el presidente colombiano con los paramilitares" (Lamrani, 2008). Este tipo de acusaciones llevan a pensar en la otra variable de Tilly en cuanto al crimen organizado del Estado, que toma medidas solo para cumplir con cuotas de resultados exigidas por sus contrapartes en la ayuda militar y contra el narcotráfico.

Esta acusación generó un fuerte impacto al presidente Álvaro Uribe, quien tuvo una reacción inmediata y acusó a Venezuela y Ecuador de financiar y mantener estrechas relaciones con las FARC. Además, el general Óscar Naranjo, director de la Policía Nacional, mostró unos documentos recuperados de la operación militar realizada en Ecuador donde se relacionaba a la guerrilla con autoridades ecuatorianas. Sin embargo, Ecuador no lo aceptó y anunció que la única persona que había tenido relación con las FARC era Gustavo Larrea, ministro de Seguridad, quien en 2007, siendo parte de la delegación internacional, participó en la liberación de Consuelo Rodríguez y Clara Rojas (Lamrani, 2008).

Luego de estas acusaciones, el ministro ecuatoriano de Relaciones Exteriores notificó la ruptura de las relaciones diplomáticas con Colombia debido a, entre otros motivos, al Plan Colombia (Veillette, 2005): "Ecuador ha sido afectado, según una organización colombiana de vigilancia de los Derechos Humanos, por un flujo de colombianos desplazados que huyen de los combates en el sur de Colombia" (p. 13). Por su parte, Venezuela también expulsó al embajador colombiano de su país luego de estos ataques y de las tensiones que se produjeron posteriormente por el secuestro "de un funcionario de las FARC en Venezuela, que luego se presentó bajo custodia colombiana" (Veillette, 2005, p. 13). 
Como se observa, el deterioro de las relaciones diplomáticas entre Colombia y sus países vecinos llegaron finalmente a una ruptura, a tal punto que Ecuador y Venezuela expulsaron los embajadores colombianos de su territorio, además de sus constantes acusaciones y amenazas. De la misma manera, sentían temor por los proyectos militares que tenían los Estados Unidos en sus territorios, como es el caso de Ecuador, donde los militares estaban preocupados por un acuerdo entre los Estados Unidos y El Salvador que permitía a la Marina de Guerra estadounidense usar el aeropuerto salvadoreño y a la Fuerza Aérea estadounidense utilizar el puerto ecuatoriano de la ciudad de Manta, el cual podría ser el lugar para un bombardeo aéreo en su país (Chomsky, 2000).

\section{Impacto internacional}

Este suceso tuvo impacto en las relaciones internacionales, lo cual se reflejó en que varios países declararon que juzgaban el acto de Colombia como una falta grave a la alteración de la soberanía de Ecuador.

Chile, con su presidenta Michelle Bachelet, castigó la acción cometida por Álvaro Uribe. La mandataria declaró: "No podemos estar de acuerdo con la violación de las fronteras, las fronteras entre los países se basan en acuerdos internacionales y no pueden violarse poco importa el objetivo, legítimo o ilegítimo" (Subcomandante Marcos, 2008). Por lo tanto, Bachelet pidió al gobierno colombiano unas disculpas a Ecuador y a los demás gobiernos latinoamericanos condenando el ataque al campamento de Raúl Reyes.

Argentina, por su parte, no aceptó la violación de soberanía por parte de Colombia y manifestó su preocupación ante el periódico argentino La Nación:

El gobierno de Cristina Kirchner expresó su 'honda preocupación' por la escalada de tensión entre Venezuela, Ecuador y Colombia, y, en una postura de fuerte impacto político, respaldó a Hugo Chávez en su acusación de que las Fuerzas Militares colombianas violaron territorio ecuatoriano en el operativo que terminó con la vida del número dos de las FARC, Raúl Reyes. (Cámara de Diputados, 2008, p. 28)

Asimismo, Brasil vio el atentado como un problema para la integración regional, y su presidente Luiz Inácio Lula da Silva tomó la decisión de hablar con los presidentes de Ecuador y Colombia para aclarar la situación, ya que existían versiones diferentes en este hecho. No suficiente con esta conversación, el canciller brasileño, Celso Amorim, propuso una comisión de investigación a la OEA para hacer una aclaración de las incertidumbres presentadas frente a los demás países (Cámara de Diputados, 2008). También se pronunció Florisvaldo Fier, vicepresidente de Mercosur, quien relacionó el ataque de Colombia con la Carta de la OEA, específicamente con en el capítulo VI, artículo 28, nombrado Seguridad Colectiva, el cual expresa: "Toda agresión de un Estado contra la integridad o la inviolabilidad del territorio o contra la 
soberanía o la independencia política de un Estado americano será considerada como un acto de agresión contra los demás Estados americanos" (OEA, 1993).

En este contexto, el 18 de marzo de 2008 se congregaron ocho países en la reunión de la Organización de Estados Americanos con la finalidad de hacer una declaración frente a la incursión de Colombia en Ecuador. Sin embargo, pese a que Ecuador suavizó su postura, al reclamar un "rechazo", en vez de una "condena" a la incursión, como exigía inicialmente, el secretario general de la OEA, José Miguel Insulza, reconoció que había "una media docena" de temas en los cuales los dos países no se habían puesto de acuerdo. De tal suerte, Insulza disolvió el pleno de cancilleres y dejó a los representantes de los ocho países para que trataran de llegar a un acuerdo y expedir una resolución final (Cámara de Diputados, 2008).

Los países que fueron parte de esta resolución eran Ecuador, Colombia, Perú, Bolivia, México, Canadá, Paraguay y República Dominicana. Por su parte, Colombia no aceptó valorar el ataque, sino que, por el contrario, advirtió con promover un plan para que los países que la estaban condenando tuvieran un mayor compromiso con la guerra contra el terrorismo y apeló al derecho a la legítima defensa, establecido por la Carta de la Organización de las Naciones Unidas (ONU) (Cámara de Diputados, 2008).

La posición de Cuba estuvo en contra del ataque y culpó principalmente a los Estados Unidos, pero aclaró que no eran enemigos de Colombia. En palabras de Fidel Castro:

El imperialismo acaba de cometer un monstruoso crimen. Fueron bombas yanquis, guiadas por satélites yanquis. Bombas mortíferas fueron lanzadas en la madrugada contra un grupo de hombres y mujeres que, casi sin excepción, dormían [...]. Las acusaciones concretas contra ese grupo de seres humanos no justifican la acción. ("Ecuador fue atacado...," 2008)

El gobierno de Nicaragua rompió relaciones diplomáticas con Colombia el 6 de marzo; sin embargo, durante la XX Cumbre del Grupo de Río se restablecieron. Prensa Latina (2008; citada por Lamrani, 2008) menciona que el gobierno mexicano y el costarricense también estaban en desacuerdo:

El presidente de México, Felipe Calderón, lanzó una llamada al diálogo, mientras que el presidente de Costa Rica, Óscar Arias, calificó el ataque de "violación de la soberanía ecuatoriana" y alertó contra "una internacionalización de un asunto delicado que es prioritariamente bilateral". Guatemala exhortó a las dos partes a la negociación. (Prensa Latina, 2008; citada por Lamrani, 2008)

Las inquietudes y preocupaciones no solamente se evidenciaron desde América Latina, sino que también fueron expresadas por países europeos, principalmente por Italia y España: "Italia expresó su preocupación mediante la voz de su canciller, Massimo d'Alema: 'Esta operación militar llevada a cabo en el exterior nos afectó mucho y nos deja perplejos y preocupa- 
dos.' España también expresó su preocupación" (Agencia Bolivariana de Noticias, 2008; citada por Lamrani, 2008). La preocupación internacional se evidenció con la oposición que se hizo al gobierno colombiano acusándolo de que era un vecino que no es de fiar porque puede violar la soberanía de sus países vecinos a través de acciones bélicas.

Por su parte, Estados Unidos ejerció su papel de proteccionista frente a su aliado, como lo evidencia el pronunciamiento de Tom Casey desde el Departamento de Estado: "Apoyamos totalmente los esfuerzos de Colombia, del presidente Uribe para responder a esta amenaza [de las FARC]" (Lamrani, 2008). El presidente Bush, a su vez, expresó su completo apoyo: "Nos oponemos con firmeza a todo acto de agresión que pudiera desestabilizar la región" (Lamrani, 2008).

\section{El apoyo de los Estados Unidos a Colombia con el TLC}

Ahora bien, como se mencionó, los Estados Unidos apoyaron a Colombia frente a su rivalidad con Ecuador, como lo expresó Bush :

"Nuestro mensaje a Colombia es que apoyamos a nuestro aliado democrático". El mandatario llamó a la unidad entre republicanos y demócratas para aprobar el Tratado de Libre Comercio, como demostración de respaldo con un aliado. "Nuestro mensaje al Congreso es que la aprobación del TLC es una cuestión de política económica sensata, y una cuestión de seguridad nacional". ("George W. Bush expresa 'completo apoyo'..., 2008)

Los tratados de libre comercio se fundamentan en la teoría neoliberal, la cual plantea que para impulsar las economías nacionales es necesario que abran sus mercados, lo cual lleva a eliminar las barreras arancelarias y facilitar la apertura de los mercados. Al respecto, Ludwig Bon Mises considera que "la economía de mercado es fundamental para organizar el intercambio que permite generar riqueza" (citado por Calderón, 2005, p. 82).

En la balanza comercial de Colombia predominan las importaciones sobre las exportaciones, lo cual hace que el gobierno deba buscar nuevos mercados que resulten eficientes y generen beneficios. En general, esto se logra a través de una gestión apropiada de las relaciones comerciales para negociar un tratado de libre comercio, como sucedió entre Colombia y Estados Unidos. Gracias a la negociación del Plan Colombia, ambos países construyeron una gran relación, así que decidieron entablar acuerdos para incrementar la producción colombiana y mejorar las exportaciones.

Las negociaciones del TLC entre Colombia y los Estados Unidos iniciaron de la siguiente manera:

mediante actas N. ${ }^{\circ} 69$ y 70 del Consejo Superior de Comercio Exterior de los días 18 de marzo y 29 de mayo de 2003, se instruyó al MCIT (Ministerio de Comercio, Industria y Turismo) comenzar la negociación con EE.UU. y se realizó una evaluación del impacto económico y social del TLC con ese país. (Mincomercio, 2020) 
Durante los meses de agosto y diciembre se realizaron reuniones en los dos países con la finalidad de acordar los temas preliminares para la negociación. Posteriormente, en noviembre de 2003, "el representante comercial de Estados Unidos anunció oficialmente la intención de su país de iniciar las negociaciones de un TLC con Colombia, Ecuador y Perú, y dejó abierto el camino para los otros países de la Comunidad Andina de Naciones" (Mincomercio, 2020). Como respuesta, el presidente de Colombia, Álvaro Uribe Vélez, defendió la necesidad del tratado comercial para incrementar la inversión extranjera en el país y a la vez lograr avances en la guerra contra el terrorismo, en este caso de las FARC, guerrilla que se ha mantenido durante un largo tiempo y es reconocida por ser la más duradera en América Latina.

Desde la perspectiva analítica de Charles Tilly (2007), el TLC sería una manera de extracción de recursos por parte de los Estados Unidos a Colombia, donde los estadounidenses tienen una influencia muy importante debido al Plan Colombia y, en consecuencia, a los grandes recursos monetarios y equipos que han suministrado para finalizar la guerra de las drogas y el terrorismo. Esta condición genera una acumulación de capital a partir de préstamos y compras realizadas por la población colombiana, lo cual constituye un claro ejemplo del postulado de Tilly (2007): "A partir de la guerra, la extracción y la acumulación de capital pueden emerger Estados nacionales" (p. 5). Estados Unidos cumplió su objetivo y le sacó provecho a la negociación del TLC, ya que Colombia no podía exigirle de la misma manera debido a la protección que estaba recibiendo.

\section{Visión estadounidense frente al Plan Colombia}

La visión de los Estados Unidos frente al Plan Colombia fue positiva, como lo señala The Washington Post (Miroff, 2016):

Demócratas y republicanos ven la ayuda de 16 años y \$10 mil millones como un gran logro de la política exterior estadounidense en el siglo XXI. Colombia, se ha convertido en su gran aliado de América del Sur, con un crecimiento de 50 millones y además un socio de libre comercio. (traducción propia)

La ayuda que ha ofrecido los Estados Unidos al gobierno colombiano ha sido gastada principalmente en seguridad. Concretamente, la intervención estadounidense produjo un giro del país hacia la guerra, lo cual generó un robustecimiento de las Fuerzas Militares colombianas y de sus instituciones para combatir de manera más eficiente los ataques de las FARC y de los carteles de narcotráfico, que cada vez tenían una mayor presencia en el territorio nacional. Las operaciones militares se lograrían a través de los sistemas de inteligencia de recolección de información que proveían los americanos, además del hardware mortal que atacaría desde las alturas a estos rebeldes y terroristas. Del mismo modo, la publicación por parte de la Organización de las Naciones Unidas evidenció los avances de este apoyo en equipo y conoci- 
miento estadounidense al gobierno colombiano, ya que se redujo el 28 \% de toda la plantación de coca en Colombia, Bolivia y Perú (Miroff, 2016).

Sin embargo, es necesario señalar que los estadounidenses también tienen una visión negativa sobre el Plan Colombia, la cual se relaciona con las Fuerzas Militares de ambos países. Específicamente, el inconformismo se debe a los abusos y la ineficacia del Ejército colombiano por su relación directa con los grupos paramilitares, lo cual vincula al Ejército norteamericano con la guerra civil colombiana, todo esto mientras el gobierno Bush seguía apoyando el Plan Colombia a través del suministro de dinero ("Looking Beyond Plan Colombia", 2001).

Aunque los paramilitares pudieron actuar como "aliado estratégico del Estado, sobre la base de diseños, políticas y estrategias institucionales que lo habilitaron" (Gutiérrez, 2015, p. 23), no se puede negar que el sistema militar y judicial colombiano realizó acciones para combatir estos grupos al margen de la ley, los cuales surgieron a partir de la insubordinación de élites rurales legales como los terratenientes, la insubordinación de élites ilegales como los narcotraficantes y la relación ideológica con políticos que tenía la finalidad de acabar con la guerrilla y con la inseguridad en el espacio rural, principalmente (Gutiérrez, 2015).

Al respecto, Russell Crandall, exasesor de la Casa Blanca, también ha expresado su duda frente a la guerra contra el narcotráfico. Con base en una revisión del Plan Colombia, Crandall sostiene que los resultados son desmoralizadores porque la cantidad de drogas y cocaína en los Estados Unidos no se ha reducido ("Looking Beyond Plan Colombia”, 2001). Este es el reflejo de la inconformidad de los Estados Unidos con los resultados, según ellos ineficaces por la poca reducción de la producción de droga y por la relación con las Fuerzas Militares colombianas, que estaban siendo acusadas por vínculos con los grupos paramilitares.

\section{Alianza militar}

La alianza militar entre Colombia y los Estados Unidos se fortaleció a través del Plan Colombia, ya que las Fuerzas Armadas colombianas no solo ampliaron su tamaño, sino que además se modernizaron, en un proceso que incluyó "el acceso a la tecnología, el armamento, la información de inteligencia y la asesoría sobre el terreno por parte de la primera potencia militar del mundo" (Rojas, 2017, p. 39). En consecuencia, las Fuerzas Armadas y de Policía colombianas se convirtieron en las mejores de Latinoamérica en lo que respecta al combate de grupos contrainsurgentes. Los Estados Unidos necesitaban que su aliado mejorara su poder militar para así cumplir de forma eficiente su objetivo de eliminar el terrorismo y el narcotráfico, además de lograr una supremacía para intimidar a sus rivales.

Respecto a esta alianza militar, el pensamiento de Charles Tilly (2007) permite reconocer el papel central que tiene la fuerza en la consolidación de los Estados nacionales. En este caso, el mejoramiento de las Fuerzas Militares colombianas logra un mayor entendimiento del desarrollo y modificación de las formas de gobierno, ya que las ocupaciones del Estado no 
solamente se basan en la amenaza de violencia, ni el único servicio que ofrece el gobierno es la protección. Dado que el uso de la fuerza tiene un costo elevado, como en este caso, donde el gasto que hace Colombia en las Fuerzas Militares es alto, los ciudadanos pueden aceptar que existan otros servicios que los beneficien y que, por lo tanto, compensen los gastos hacia aquel monopolio de la violencia (Tilly, 2007).

El fortalecimiento de las Fuerzas Militares logró que Colombia se posicionara notoriamente frente a los grupos subversivos, a la vez que mostró que la forma de gobierno estaba consolidada firmemente y que podía proteger a toda la población. Esta necesidad de avanzar responde a la constante amenaza de violencia que generan el narcotráfico y las FARC, las cuales fueron obligadas a realizar diálogos de paz en el siguiente periodo presidencial de Juan Manuel Santos, quien se negó a acabar definitivamente con esta guerrilla en el plano militar.

Desde que inició la implementación del Plan Colombia, entre los años 2009 y 2013 las Fuerzas Militares contaron con una estrategia enfocada hacia el control del territorio, además de tener una presencia del gobierno y atender a la población vulnerable, que había sido la más afectada por el conflicto interno. Aunque cabe aclarar que no solamente bastaba con la presencia militar, ya que

la recuperación permanente del control territorial sería posible solo si se consolidaba la presencia del Estado en todas sus dimensiones, y no únicamente en la militar; ello incluía satisfacer las necesidades básicas de la población, tales como salud, educación, servicios públicos y justicia, y proyectos de infraestructura. (Rojas, 2017, p. 41)

Ahora bien, dado que una de las funciones esenciales del Estado es proteger el territorio y sus habitantes, la primera acción de las Fuerzas Militares fue recuperar su dominio territorial y, posteriormente, atender a la población frente a todas sus necesidades primarias.

Por otro lado, una muestra del apoyo de Colombia hacia los Estados Unidos fue su ayuda a la campaña de Irak, donde los estadounidenses hicieron una práctica diferente a la normatividad internacional en que se habían mantenido, dentro de las soluciones pacíficas, o la aprobación de inmunidad jurídica para estos ciudadanos frente al Tribunal Penal Internacional (Rojas, 2006). Esto evidencia que Colombia se puso en un papel de coerción cuando recibió la ayuda de los Estados Unidos, ya que al ser protegido por un actor que tiene tanto poder, debe responderle con el mismo apoyo sin importar que no le sea beneficioso.

Una muestra de la coerción se refleja cuando tropas colombianas apoyaron a los Estados Unidos en la guerra contra los talibanes en Afganistán en el año 2009. Este apoyo de Colombia muestra el alineamiento militar que se forjó por la financiación que recibió el país en el Plan Colombia, además del entrenamiento y la capacitación por parte de las Boinas Verdes, quienes "ayudaron a entrenar a una unidad de Operaciones Especiales de la Policía conocida como 'Comandos de la Selva'. Los comandos alcanzaron objetivos en lo profundo de la jun- 
gla, destruyendo laboratorios de drogas y eliminando a los principales capos de las drogas" ("Colombia to aid U.S...", 2009). Este acto forma parte de la monopolización de la violencia que se necesita para hacer un Estado más poderoso y rico, como claramente lo han hecho los Estados Unidos a lo largo de sus historia a partir de las guerras y las alianzas que han establecido con Estados estratégicos a los que les puedan sacar un beneficio propio.

La alianza militar fue importante para Colombia debido a los logros que tuvo en controlar a las FARC, que se redujeron de veinte mil hombres iniciales a diez mil hombres, además del progreso en la capacidad de respuesta ante un enfrentamiento que tuvieron las Fuerzas Armadas y de Policía colombianas. Tanto es así que hasta la actualidad Colombia exporta su conocimiento táctico-militar en el enfrentamiento del narcotráfico y el terrorismo a otros países como Afganistán, Uruguay, Paraguay, República Dominicana, Trinidad y Tobago, Jamaica, México y Haití, lo cual demuestra que el gobierno colombiano no es un Estado fallido, sino que, por el contrario, es un Estado con porvenir (De la Torre, 2010).

Por lo tanto, se puede afirmar que Colombia obtuvo grandes beneficios en la alianza militar con los Estados Unidos, lo cual se refleja en su creciente conocimiento estratégico y en el incremento y uso de armamento, a tal punto que ahora exporta sus aprendizajes y experiencia a otros países latinoamericanos. En definitiva, esto evidencia que la alianza militar fue provechosa no solo para Colombia y los Estados Unidos, sino también para los otros países que están optimizando su estrategia militar.

\section{Conclusiones}

Respecto al impacto que tuvo la ayuda internacional de Estados Unidos a través del Plan Colombia en las relaciones internacionales entre Colombia y los países vecinos, se evidencia que las relaciones internacionales del país tuvieron un viraje y profundizaron su dependencia con los Estados Unidos en detrimento de otras alianzas más cercanas debido a la coyuntura ideológica que atravesaba Latinoamérica en ese momento, basada en gobiernos demasiado liberales.

Este cambio ocasionó una enemistad con los gobiernos vecinos debido a que desconfiaban de esta alianza de Colombia con la potencia mundial y, por lo tanto, tenían un punto de vista negativo: "Otras cosas piensan las autoridades fronterizas que vieron al Plan como un monstruo de mil cabezas que puede involucrarlos en un mediano plazo en el complejo conflicto colombiano" (El Tiempo, 2000). Se pensaba que los cultivos podrían cruzar las fronteras hacia Ecuador, Brasil, Perú y Venezuela, y que llevarían consigo un desplazamiento masivo por confrontación armada, lo cual planteaba un riesgo para la seguridad nacional de los países vecinos.

En este contexto, Colombia tuvo una ruptura de sus relaciones con Ecuador cuando el 29 de febrero de 2008 sus Fuerzas Militares y de Policía invadieron el territorio ecuatoriano para bombardear un campamento, en el cual murió Raúl Reyes, uno de los comandantes de 
las FARC. Esta acción impactó las relaciones internacionales con varios países, que manifestaron unánimemente a través de diversas declaraciones que juzgaban el acto de Colombia como una falta grave a la soberanía de Ecuador. Asimismo, la mala relación que tenía con Venezuela empeoró significativamente, a tal punto que se acabaron las relaciones diplomáticas que tenían los dos gobiernos. Sin embargo, también se debe señalar que varios países latinoamericanos sacaron provecho del conocimiento que las Fuerzas Militares colombianas habían adquirido gracias a su alianza militar con los Estados Unidos, pues importaron varias de estas instrucciones y estrategias con el objetivo de fortalecer sus propias fuerzas armadas.

Así, con base en la propuesta de Charles Tilly (2007), se concluye que la ayuda internacional de los Estados Unidos tuvo desde un inicio el interés de hacer una extracción de recursos a partir del Tratado de Libre Comercio, como efectivamente sucedió, pues el Estado colombiano estaba en desventaja para negociar los términos del acuerdo debido a la ayuda que había recibido, así que no tuvo otro camino que aceptar. En este sentido, el análisis muestra que los Estados Unidos tienen un papel de protección, en donde "elimina y neutraliza a los enemigos de sus clientes" (Tilly, 2007), en este caso, Colombia. Asimismo, se evidencia que el gobierno estadounidense tomó provecho al formar y fortalecer la alianza militar con Colombia, ya que esto le proporcionó poderío militar en el país para intimidar a los países vecinos. Esto corresponde, en palabras de Tilly (2007), a neutralizar "a los rivales fuera del territorio en el cual tienen preferencia permanente y notoria en el uso de la fuerza" (p. 17). Por lo tanto, respecto a la noción de construcción del Estado, se puede afirmar que los Estados Unidos impusieron su supremacía a la soberanía de Colombia por medio del poder que tenían sobre la situación que atravesaba el país en este momento.

Finalmente, se concluye que Colombia debilitó sus relaciones internacionales cuando decidió plegarse a los Estados Unidos como único aliado estratégico. En ese momento, el gobierno estadounidense tenía una agenda en contra del terrorismo talibán, de manera que el país acogió los términos de terrorismo en la lucha del Estado contra la delincuencia, el narcotráfico y los grupos al margen de la ley. Precisamente, esta vuelve a ser hoy la amenaza en materia internacional para Colombia, pues el gobierno del presidente Iván Duque, asesorado por el expresidente Uribe, se ha plegado nuevamente a los Estados Unidos y ha desconocido los tratados y las alianzas con otros gobiernos en otras latitudes. El riesgo consiste en que estas alianzas a las que renuncia el gobierno colombiano son las que pueden evitar la dependencia y la extracción por parte de un solo aliado, el cual ofrece su ayuda a cambio de un cheque en blanco condicionado y dependiente.

\section{Agradecimientos}

Los autores desean agradecer a la Universidad de La Salle por su apoyo en la realización de este artículo. 


\section{Declaración de divulgación}

Los autores declaran que no existe ningún potencial conflicto de interés relacionado con el artículo.

\section{Financiamiento}

Los autores no declaran fuente de financiamiento para la realización de este artículo.

\section{Sobre los autores}

Robert Manuel Ojeda Pérez es Ph.D. en Educación y Sociedad de la Universidad de La Salle, magíster en Historia de la Universidad de Los Andes, especialista en Pedagogía y Didáctica, e historiador de la Pontificia Universidad Javeriana. Es miembro de número de la Academia de Historia de Bogotá.

https://orcid.org/0000-0002-1227-7854 - Contacto: rojeda@unisalle.edu.co

Elizabeth Martínez Cruz es estudiante de Negocios y Relaciones Internacionales en la Universidad de La Salle Bogotá, D. C., Colombia. Investigadora del Semillero Se-Koiné de la misma institución.

https://orcid.org/0000-0003-0110-092X - Contacto: emartinez17@unisalle.edu.co

\section{Referencias}

Aranguren, D. (2013). Análisis de los efectos del Plan Colombia en la configuración de la agenda de seguridad nacional de Ecuador [tesis de pregrado, Universidad del Rosario]. Repositorio Institucional. http://repository.urosario.edu.co/bitstream/handle/10336/4956/1018441756-2013.pdf

Calderón, I. (2005). La teoría neoliberal y la globalización [tesis de licenciatura, Universidad de las Américas Puebla]. Repositorio Institucional. http://catarina.udlap.mx/u_dl_a/tales/documentos/lri/calderon_m_is/capitulo2.pdf

Cámara de Diputados. (2008). Anexo. El Universal. http://www.diputados.gob.mx/sedia/sia/spe/SPEISS-08-08_Anexo-Universal.pdf

Chomsky, N. (2000) Plan Colombia. Innovar Revista de Ciencias Administrativas y Sociales, 16, 9-26. https:// revistas.unal.edu.co/index.php/innovar/article/view/24370/24974

Colombia to aid U.S. in taliban fight. (2009, julio 27). CBS. https://www.cbsnews.com/news/colombia-toaid-us-in-taliban-fight/

Cortés, V., \& Águila C. (2012). Crisis Colombia-Ecuador. Revista C+R AE-IC Tarragona. http://www.aeic2012tarragona.org/comunicacions_cd/ok/369.pdf

De la Torre, V. (2010). Plan Colombia: 10 años después. El Espectador. https://www.elespectador.com/ impreso/internacional/articuloimpreso-213835-plan-colombia-diez-anos-despues

Ecuador fue atacado con bombas de EE. UU. guiadas por satélites "yanquis", dice Fidel Castro. (2008, marzo 4). Caracol Radio. https://caracol.com.co/radio/2008/03/04/nacional/1204626720_558271. html

García, L. (2013). El narcotráfico en Colombia: de las falencias de la política de la prohibición y sus secuelas, a la discusión de la discriminación y despenalización [tesis de maestría, Pontificia Universidad Javeriana]. Repositorio Institucional. https://repository.javeriana.edu.co/bitstream/handle/10554/15289/GarciaLunaGonzaloErnesto2013.pdf 
George W. Bush expresa "completo apoyo" a Álvaro Uribe en conflicto diplomático con vecinos. (2008, marzo 5). El Tiempo. https://www.eltiempo.com/archivo/documento/CMS-3985275

Gutiérrez, F. (2015) ¿Una historia simple? https://www.ambitojuridico.com/BancoMedios/Documentos\%20PDF/una-historia-simple-1447167162-1460380556(1).pdf

Lamrani, S. (2008). George W. Bush y Álvaro Uribe contra la paz en Colombia. Rebelión. Recuperado de: https://rebelion.org/george-w-bush-y-alvaro-uribe-contra-la-paz-en-colombia/

Looking Beyond Plan Colombia. (2001, mayo 18). The New York Times. https://www.nytimes. com/2001/05/18/opinion/looking-beyond-plan-colombia.html

Mincomercio. (2020). Tratados de Libre Comercio. http://www.tlc.gov.co/acuerdos/vigente/acuerdo-de-promocion-comercial-entre-la-republ-1/contenido/resumen-del-acuerdo

Miroff, N. (2016, septiembre 18). 'Plan Colombia': How Washington learned to love Latin American intervention again. The Washington Post. https://www.washingtonpost.com/world/the_americas/ plan-colombia-how-washington-learned-to-love-latin-american-intervention-again/2016/09/18/ ddaeae1c-3199-4ea3-8d0f-69ee1cbda589_story.html?noredirect=on\&utm_term=.dfbf757a3cd8

Moreano, U. (2005). Colombia y sus vecinos frente al conflicto armado. Ediciones Abya-Yala.

Organización de Estados Americanos [OEA]. (1993). Carta de la Organización de los Estados Americanos. http://www.oas.org/es/sla/ddi/tratados_multilaterales_interamericanos_A-41_carta_OEA.asp

Rojas, M. (2006). Balance de la política internacional del gobierno de Uribe. Análisis Político, 57, 85-105. http://www.scielo.org.co/pdf/anpol/v19n57/v19n57a05.pdf

Rojas, M. (2017). Estados Unidos en la construcción de la paz en Colombia. Revista Análisis Político, 91, 37-52. https://revistas.unal.edu.co/index.php/anpol/article/view/70263/64690

Subcomandante Marcos. (2008, marzo 4). Chile condena violación de Colombia a la soberanía de Ecuador. Rebelión. https://rebelion.org/chile-condena-violacion-de-colombia-a-la-soberania-de-ecuador/

Tickner, B. (2008). Colombia y Estados Unidos: una relación "especial". Foreign Affairs, 8(4), http://revistafal. com/numeros-anteriores/fal-08-4/

Tilly, C. (2007). Guerra y construcción del Estado como crimen organizado. Revista Académica de Relaciones Internacionales, 5. https://revistas.uam.es/index.php/relacionesinternacionales/article/ view/4866/5335

Veillette, C. (2005). Plan Colombia: A Progress Report. CRS Report for Congress. https://fas.org/sgp/crs/ row/RL32774.pdf

Venezuela y Colombia: años en la cuerda floja. (2010, julio 23). BBC Mundo. https://www.bbc.com/mundo/ america_latina/2010/07/100722_cronologia_relaciones_venezuela_colombia_Ir 